\title{
Asymptotic behavior of stochastic $p$-Laplacian-type equation with multiplicative noise
}

Wenqiang Zhao*

"Correspondence:
zhaowq.ctbu@gmail.com
School of Mathematics and
Statistics, Chongqing Technology
and Business University, Chongqing
400067, China

400067, China

\begin{abstract}
The unique existence of solutions to stochastic $p$-Laplacian-type equation with forced term satisfying some growth and dissipative conditions is established for the initial value in $L^{2}(D)$. The generation of a continuous random dynamical system and the existence of a random attractor for stochastic $p$-Laplacian-type equation driven by multiplicative noise are obtained. Furthermore, we obtain a random attractor consisting of a single point and thus the system possesses a unique stationary solution.
\end{abstract}

MSC: $60 \mathrm{H} 15 ; 35 \mathrm{~B} 40 ; 35 \mathrm{~B} 41$

Keywords: random dynamical systems; stochastic $p$-Laplacian-type equation; random attractors

\section{Introduction}

The purpose of this paper is to investigate the long-time behavior of solutions to stochastic $p$-Laplacian-type equation with multiplicative noise, which reads

$$
\begin{aligned}
& d u+\left(\triangle \Phi_{p}(\triangle u)+g(x, u)\right) d t=f(x) d t+b u \circ d W(t), \\
& u(x, 0)=u_{0}, \quad x \in D, \\
& \left.\triangle u(t)\right|_{\partial D}=0,\left.\quad u(t)\right|_{\partial D}=0,
\end{aligned}
$$

where $\Phi_{p}(s)=|s|^{p-2} s, p \geq 2$; $D$ is an open and bounded subset of $\mathbb{R}^{n}$ with regular boundary $\partial D$; $\Delta$ is the Laplacian with regard to the variable $x \in D ; b$ is a positive constant; $u(t)=$ $u(x, t)$ a real-valued variable of $x \in D, t \geq 0$; $W(t)$ is mutually independent two-sided realvalued Wiener process defined on a complete probability space $(\Omega, \mathcal{F}, \mathbb{P})$, where

$$
\Omega=\{\omega \in C(\mathbb{R}, \mathbb{R}): \omega(0)=0\}
$$

and $\mathcal{F}$ is the Borel $\sigma$-algebra induced by the compact-open topology of $\Omega$, and $\mathbb{P}$ is the corresponding Wiener measure on $(\Omega, \mathcal{F})$. Then we can identify $W(t)$ with $\omega(t)$

$$
W(t)=\omega(t), \quad t \in \mathbb{R} .
$$

@ 2012 Zhao; licensee Springer. This is an Open Access article distributed under the terms of the Creative Commons Attribution License (http://creativecommons.org/licenses/by/2.0), which permits unrestricted use, distribution, and reproduction in any medium, provided the original work is properly cited. 
It is known that the random attractor, which characterizes the long-time behavior of random dynamical systems (RDS) perfectly, was first introduced by $[6,13]$ as a generalization of a global attractor for deterministic PDE. The existences of the random attractor for RDS have been richly developed by many authors for all kinds of SPDEs, see [2, 5, 6, $9,10,15-18,21-25]$ and references therein.

In deterministic case, there is a large number of works about the $p$-Laplacian-type equation. Temam [14] obtained the global attractor for (1.1) with exterior forcing term $g(x, u)=k u$, a simple case. In recent years, Yang et al. $[19,20]$ considered the global attractor for a general $p$-Laplacian-type equation defined both on unbounded domain and bounded domain, respectively. The uniform attractor was also investigated by Chen and Zhong [3] in nonautonomous case. In random case, Zhao [23] obtained random attractors for the $p$-Laplacian-type equation driven by additive noise.

In this paper, we consider the existence of a random attractor for (1.1)-(1.3) with exterior forcing term $g(x, u)$ satisfying some growth conditions. The multiplicative noise $c u \circ d W(t)$ characterizes, to some extent, some of the minimal fluctuations among environment or a man-made complex system, which we should take into consideration in order to model perfectly the concrete problem.

One difficulty in our discussions is to estimate the solution operator in the stronger norm space $V$, where $V \subset H \subset V^{\prime}$ is the Gelfand triple, see Section 2. It seems that the methods used in unperturbed case (see $[14,19,20])$ are completely unavailable because of the leading term $\triangle \Phi_{p}(\Delta u)$ with high order differentials and the forcing term $g(x, u)$ with $p-1$ times growth.

We need to develop some techniques to surmount the obstacle, though we also follow the classic approach (based on the compact embedding) widely used in [5, 6, 17, 21-24] and so on. By using the properties of Dirichlet form for the Laplacian, we overcome this obstacle and obtain the estimate of the solution in the Sobolev space $V_{0}$, which is weaker than $V$. Here some basic results about the Laplacian are used. We refer to [8] to obtain the details on Dirichlet forms for a negative definite and self-adjoint operator. The existence and uniqueness of a continuous RDS are proved by employing the standard in [12].

We give the outline of this paper. In Section 2, we present some preliminaries for the theory of RDS and the results about the Laplacian which are necessary to our discussion. In Section 3, we prove the existence and uniqueness of a continuous RDS which is generated by the solution to stochastic $p$-Laplacian-type equation with multiplicative noise. In Section 4, we give some estimates for the solution operators in given Hilbert space and then obtain a random attractor for this RDS. In the last part, we show that the system possesses a unique stationary point under a given condition.

\section{Preliminaries}

In this section, we present some basic notions about RDS, which can be found in $[1,4-6]$. We also list the Sobolev spaces, some results about the Laplacian and its Dirichlet forms.

The basic notion in RDS is a metric dynamical system (MSD) $\theta \equiv\left(\Omega, \mathcal{F}, \mathbb{P},\left\{\theta_{t}\right\}_{t \in \mathbb{R}}\right)$, which is a probability space $(\Omega, \mathcal{F}, \mathbb{P})$ with a group $\theta_{t}, t \in \mathbb{R}$, of measure preserving transformations of $(\Omega, \mathcal{F}, \mathbb{P})$. MSD $\theta$ is said to be ergodic under $\mathbb{P}$ if for any $\theta$-invariant $\operatorname{set} B \in \mathcal{F}$ we have either $\mathbb{P}(B)=0$ or $\mathbb{P}(B)=1$, where the $\theta$-invariant set is in the sense $\theta_{t} B=B$ for $B \in \mathcal{F}$ and all $t \in \mathbb{R}$.

RDS is an object consisting of a MSD and a cocycle over this MSD, where the MSD is used to model random perturbations. Let $X$ be complete and separable metric space with 
metric $d$ and Borel sigma-algebra $B(X)$, i.e., the smallest $\sigma$-algebra on $X$ which contains all open subsets.

Definition 2.1 (1) A continuous RDS on $(X, d)$ over a MSD $\theta$ is a family of measurable mappings

$$
\varphi: \mathbb{R}^{+} \times \Omega \times X \rightarrow X, \quad(t, \omega, x) \mapsto \varphi(t, \omega) x
$$

such that for $\mathbb{P}$-a.e. $\omega \in \Omega$, the mappings $\varphi(t, \omega)$ satisfy the cocycle property

$$
\varphi(0, \omega)=i d, \quad \varphi(t+s, \omega)=\varphi\left(t, \theta_{s} \omega\right) \varphi(s, \omega)
$$

for all $s, t \in \mathbb{R}^{+}$, and the mappings $\varphi(t, \omega): x \mapsto \varphi(t, \omega) x$ are continuous in $X$ for all $t \in \mathbb{R}^{+}$.

(2) A continuous stochastic flow is a family of measurable mappings $S(t, s ; \omega): X \rightarrow X$, $-\infty \leq s \leq t \leq \infty$, such that for $\mathbb{P}$-a.e. $\omega \in \Omega$,

$$
\begin{aligned}
& S(t, r ; \omega) S(r, s ; \omega) x=S(t, s ; \omega) x, \quad x \in X, \\
& S(t, s ; \omega) x=S\left(t-s, 0 ; \theta_{s} \omega\right) x, \quad x \in X
\end{aligned}
$$

for all $s \leq r \leq t$, and $x \mapsto S(t, s ; \omega) x$ are continuous in $X$ for all $s \leq t$.

(3) A random compact set $\{K(\omega)\}_{\omega \in \Omega}$ is a family of compact sets indexed by $\omega$ such that for every $x \in X$ the mapping $\omega \mapsto d(x, K(\omega))$ is measurable with respect to $\mathcal{F}$.

(4) A random set $\{\mathcal{A}(\omega)\}_{\omega \in \Omega}$ is an attracting set if for every deterministic bounded subset $B \subset X$ and $\mathbb{P}$-a.e. $\omega \in \Omega$,

$$
\lim _{t \rightarrow \infty} \operatorname{dist}\left(\varphi\left(t, \theta_{-t} \omega\right) B, \mathcal{A}(\omega)\right)=0
$$

where $\operatorname{dist}(\cdot, \cdot)$ is $\operatorname{defined}$ by $\operatorname{dist}(A, B)=\sup _{x \in A} \inf _{y \in B} d(x, y)$.

(5) A random set $\{\mathcal{A}(\omega)\}_{\omega \in \Omega}$ is an absorbing set if for every deterministic bounded subset $B \subset X$ and $\mathbb{P}$-a.e. $\omega \in \Omega$, there exists $t_{B}(\omega)>0$ such that for all $t \geq t_{B}(\omega)$,

$$
\varphi\left(t, \theta_{-t} \omega\right) B \subset \mathcal{A}(\omega)
$$

where $\varphi\left(t, \theta_{-t} \omega\right) B=\bigcup_{x \in B} \varphi\left(t, \theta_{-t} \omega\right) x$.

It is obvious that an absorbing set is an attracting set. The attraction in the definition of the attracting set is a form of pathwise convergence. In fact, the attracting set also attracts in the weaker convergence in probability, in the sense, for all $\varepsilon>0$ and every bounded set $B \subset X$,

$$
\lim _{t \rightarrow \infty} \mathbb{P}\left(\operatorname{dist}_{X}\left(\varphi\left(t, \theta_{-t} \omega\right) B, \mathcal{A}(\omega)\right)>\varepsilon\right)=0 .
$$

Definition 2.2 A random compact set $\omega \mapsto \mathcal{A}(\omega)$ is called to be a random attractor for the RDS $\varphi$ if $\{\mathcal{A}(\omega)\}_{\omega \in \Omega}$ is an attracting set and $\varphi(t, \omega) \mathcal{A}(\omega)=\mathcal{A}\left(\theta_{t} \omega\right)$ for $\omega \in \Omega$ and all $t \geq 0$. 
Theorem 2.3 (see [4]) Assume that $\varphi(t, \omega)$ is a continuous $R D S$ on $X$ over MDS $\theta$. If there exists a compact random absorbing set $\{K(\omega)\}_{\omega \in \Omega}$, then $\varphi(t, \omega)$ possesses a random attractor $\{\mathcal{A}(\omega)\}_{\omega \in \Omega}$ defined by

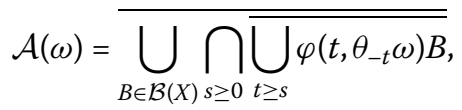

where $\mathcal{B}(X)$ denotes all the bounded subsets of $X$.

Let $L^{p}(D)$ be the $p$-times integrable functions space on $D$ with norm denoted by $\|\cdot\|_{p}$, and $V=W_{0}^{2, p}(D)$ with Sobolev equivalent norm (see p.166 of [14])

$$
\|v\|_{V}=\|\Delta v\|_{p}=\left(\int_{D}|\Delta v|^{p} d x\right)^{\frac{1}{p}}, \quad v \in V
$$

Put the dual $V^{\prime}$ of $V$ by $V^{\prime}=W^{-2, p^{\prime}}(D)$, where

$$
u \in W^{-2, p^{\prime}}(D) \Leftrightarrow u=\sum_{|\alpha| \leq 2} D^{\alpha} f_{\alpha}, \quad f_{\alpha} \in L^{p^{\prime}}(D)
$$

and $\frac{1}{p}+\frac{1}{p^{\prime}}=1$. Let $H=L^{2}(D)$ with the usual scalar product and norm $\left\{(\cdot, \cdot),\|\cdot\|_{2}\right\}$. Then we have the following Gelfand triple

$$
V \subset H \equiv H^{\prime} \subset V^{\prime},
$$

or concretely

$$
W_{0}^{2, p}(D) \subset L^{2}(D) \subset W^{-2, \frac{p}{p-1}}(D)
$$

where the injections are continuous and each space is dense in the following one.

We know that the Laplacian $\Delta$, which is negative definite and self-adjoint, is the generator (with domain $W_{0}^{2, p}(D)$ ) of a strongly continuous semigroup $M(t)$ on $L^{p}(D)$ which is contractive and positive. Here "contractive" means $\|M(t)\|_{p} \leq 1$ and "positive" means $M(t) u \geq 0$ for every $0 \leq u \in L^{p}(D)$. The resolvent of generator $\Delta$ is denoted by $R(\lambda, \Delta)$, $\lambda \in \rho(\Delta)$, where $\rho(\Delta)$ is the resolvent set of $\Delta$. By the Lumer-Phillips Theorem in [11], it follows that $(0, \infty) \subset \rho(\Delta)$ and for $u \in L^{p}(D)$

$$
\|\lambda R(\lambda, \Delta) u\|_{p} \leq\|u\|_{p}, \quad \lambda>0
$$

Moreover, for $u \in D(\Delta), R(\lambda, \Delta) u \in D(\Delta)$ and $\Delta R(\lambda, \Delta) u=R(\lambda, \Delta) \Delta u$, where $D(\Delta)$ is the domain of $\Delta$.

Since $\Delta$ is negative definite and self-adjoint, then $\Delta$ is associated with the Dirichlet forms $\mathscr{E}$ by

$$
\mathcal{E}(u, v)=(\sqrt{-\Delta} u, \sqrt{-\Delta} v), \quad u, v \in H_{0}^{1}(D) \cap H^{2}(D) .
$$


$\mathcal{E}$ is unique determined by $\Delta$. For $u, v \in H_{0}^{1}(D) \cap H^{2}(D)$, we define a new inner product by

$$
\mathcal{E}^{(\lambda)}(u, v)=\lambda(u-\lambda R(\lambda, \Delta) u, v), \quad \lambda>0,
$$

where $R(\lambda, \Delta)$ is the resolvent of $\Delta$. Then it follows from Ref. [8] that $\mathcal{E}^{(\lambda)}(u, v) \uparrow$ as $\lambda \rightarrow \infty$, and

$$
\lim _{\lambda \rightarrow \infty} \mathcal{E}^{(\lambda)}(u, v)=\varepsilon(u, v)
$$

for $u, v \in H_{0}^{1}(D) \cap H^{2}(D)$.

\section{Existence and uniqueness of RDS}

In this section, we show the existence and uniqueness of a continuous RDS for the following stochastic $p$-Laplacian-type equation with multiplicative noise,

$$
\begin{aligned}
& d u+\left(\Delta \Phi_{p}(\Delta u)+g(x, u)\right) d t=f(x) d t+b u \circ d W(t), \\
& u(x, 0)=u_{0}, \quad x \in D, \\
& \left.\Delta u(t)\right|_{\partial D}=0,\left.\quad u(t)\right|_{\partial D}=0,
\end{aligned}
$$

where $\Phi_{p}(s)=|s|^{p-2} s, p \geq 2$. To study System (3.1)-(3.3), we assume that the nonlinearity $g(x, u)$ defined in $D \times \mathbb{R}$ satisfies the following conditions:

$$
\begin{aligned}
& g(x, u) u \geq k_{1}|u|^{q}-\phi_{1}(x), \quad \phi_{1} \in L^{1}(D), k_{1} \in \mathbb{R}^{+}, \\
& |g(x, u)| \leq k_{2}|u|^{q-1}+\phi_{2}(x), \quad \phi_{2} \in L^{\frac{q}{q-1}}(D), k_{2} \in \mathbb{R}^{+}, \\
& \left(g\left(x, u_{1}\right)-g\left(x, u_{2}\right)\right)\left(u_{1}-u_{2}\right) \geq k_{3}\left|u_{1}-u_{2}\right|^{2}, \quad k_{3} \in \mathbb{R},
\end{aligned}
$$

where $2 \leq q \leq p<\infty$.

For $\omega \in \Omega$, we define a nonlinear operator $A$ on $V$ by

$$
A(u(t), \omega)=\Delta \Phi(\Delta u(t))+g(x, u(t))-f(x), \quad u(t) \in V, x \in D .
$$

Then (3.1) reads

$$
\frac{d u(t)}{d t}+A(u(t), \omega)=b u(t) \circ d W(t)
$$

Since $p \geq q$, by our assumption (3.4)-(3.6) and $f \in V^{\prime}$, it is easy to check that for given $\omega \in \Omega$, the operator $A: u \mapsto A(u, \omega)$ mapping $W^{2, p}(D)$ into $W^{-2, p^{\prime}}(D)$ is well defined, where $p^{\prime}=\frac{p}{p-1}$.

Let $(\Omega, \mathcal{F}, \mathbb{P})$ be the probability space as in the introduction. Define the Wiener shift by

$$
\theta_{t} \omega(s)=\omega(s+t)-\omega(t), \quad \omega \in \Omega, t, s \in \mathbb{R} .
$$

Then $\theta=\left(\Omega, \mathcal{F}, \mathbb{P},\left\{\theta_{t}\right\}_{t \in \mathbb{R}}\right)$ is an ergodic MDS. 
In order to obtain the existence of a continuous RDS, it is necessary to translate (3.1)-(3.3) into a deterministic system parameterized by $\omega$. To this end, we consider the Ornstein-Uhlenbeck process. Put

$$
t \mapsto z\left(\theta_{t} \omega\right):=-\int_{-\infty}^{0} e^{\tau}\left(\theta_{t} \omega\right)(\tau) d \tau, \quad t \in \mathbb{R}
$$

which solves the Itô differential equation

$$
d z\left(\theta_{t} \omega\right)+z\left(\theta_{t} \omega\right) d t=d W(t), \quad z(-\infty)=0,
$$

where the Ornstein-Uhlenbeck constant equals to 1.

Note that $z\left(\theta_{t} \omega\right)$ is a Gaussian process with mathematical expectation $\mathbb{E}\left[z\left(\theta_{t} \omega\right)\right] \equiv 0$ and variance $\sigma^{2}(t)=\frac{1}{2}$, see [7], whereas $\lim _{t \rightarrow \infty} \frac{1}{t} \int_{0}^{t} z\left(\theta_{\tau} \omega\right) d \tau=0$. Furthermore, from [2, 15, 18 ], the random variable $|z(\omega)|$ is continuous in $t$ for $\mathbb{P}$-a.e. $\omega \in \Omega$ and grows sublinearly, i.e., $\lim _{t \rightarrow \pm \infty} \frac{\left|z\left(\theta_{t} \omega\right)\right|}{|t|}=0$.

We now translate (3.1) by one classical change of variables

$$
v(t)=e^{-b z\left(\theta_{t} \omega\right)} u(t)
$$

Then we have

$$
d u(t)=e^{b z\left(\theta_{t} \omega\right)} d v(t)-b e^{b z\left(\theta_{t} \omega\right)} z\left(\theta_{t} \omega\right) v(t) d t+b u(t) \circ d W(t) .
$$

Then, formally, the variable $v(t)$ satisfies the following equations parameterized by $\omega \in \Omega$ but without white noise:

$$
\begin{aligned}
& \frac{d v}{d t}+e^{b(p-2) z\left(\theta_{t} \omega\right)} \Delta \Phi(\Delta v)+e^{-b z\left(\theta_{t} \omega\right)} g\left(x, e^{b z\left(\theta_{t} \omega\right)} v\right)=e^{-b z\left(\theta_{t} \omega\right)} f(x)+b z\left(\theta_{t} \omega\right) v, \\
& v(s)=e^{-b z\left(\theta_{s} \omega\right)} u(s), \quad x \in D, s \in \mathbb{R}, \\
& \Delta v(t)=0, \quad v(t)=0, \quad x \in \partial D, t \geq s,
\end{aligned}
$$

where $g(x, u)$ satisfies (3.4)-(3.6) and $f$ is given in $V^{\prime}, 2 \leq q \leq p<\infty$.

For convenience, we put

$$
\begin{aligned}
\bar{A}(v(t), \omega)= & e^{b(p-2) z\left(\theta_{t} \omega\right)} \Delta \Phi(\Delta v)+e^{-b z\left(\theta_{t} \omega\right)} g\left(x, e^{b z\left(\theta_{t} \omega\right)} v\right) \\
& -e^{-b z\left(\theta_{t} \omega\right)} f(x)-b z\left(\theta_{t} \omega\right) \nu
\end{aligned}
$$

Then we have

$$
\frac{d v(t)}{d t}+\bar{A}(v(t), \omega)=0
$$

Note that System (3.1)-(3.3) and System (3.10)-(3.12) are equivalent by (3.9). Let $u\left(t, \omega ; s, u_{0}\right)$ and $v\left(t, \omega ; s, v_{0}\right)$ be the solution of System (3.1)-(3.3) and System (3.10)-(3.12) respectively. It is easy to check that if System (3.1)-(3.3) possess a unique solution in $V$ for all initial values in $H$ then System (3.10)-(3.12) possess a unique solution in $V$ for the same 
initial value in $H$. Moreover, if the mapping $v_{0} \mapsto v\left(t, \omega ; s, v_{0}\right)$ is continuous in $H$ for the initial value in $H$, then the mapping $u_{0} \mapsto u\left(t, \omega ; s, u_{0}\right)$ is also continuous in $H$, vice verse.

We now show the existence and uniqueness of solution to System (3.1)-(3.6).

Theorem 3.1 Assume that $g$ satisfies (3.4)-(3.6) and $f$ is given in $V^{\prime}, 2 \leq q \leq p<\infty$. Then for all $u_{0} \in H$ with $u_{0}=u(s)$, System (3.1)-(3.3) has a unique solution

$$
u\left(t, \omega ; s, u_{0}\right) \in L_{l o c}^{p}([s, \infty), V) \cap C([s, \infty), H)
$$

for all $t \geq s$ and $\mathbb{P}$-a.e. $\omega \in \Omega$. Furthermore, the mapping $u_{0} \mapsto u\left(t, \omega ; s, u_{0}\right)$ from $H$ into $H$ is continuous for all $t \geq s$.

Proof We first show that for every $u_{0} \in H$ there exists a unique solution $u\left(t, \omega ; s, u_{0}\right) \in$ $L_{l o c}^{p}([s, \infty), V)$. By Theorem 4.2.4 and Exercise 4.1.2 in [12], it suffices to show that for every fixed $\omega \in \Omega, A(u(t), \omega)$ possesses Hemi-continuity, Monotonicity, Coercivity, and Bounded-ness properties (for the definitions of these notions please refer to p.56 of [12]). But the proofs are an analogy of the corresponding works in [23]. So we omit them here.

We then show that the solution is in $C([s, T], H)$. By our assumptions that $p \geq q$ and $f \in V^{\prime}$, we can check that $\bar{A}(v(t), \omega)$ maps $L^{p}([s, T], V)$ to $L^{p^{\prime}}\left([s, T], V^{\prime}\right)$ for $\omega \in \Omega$. Thus if $v \in$ $L^{p}([s, T], V)$, then (3.14) implies that $\frac{d v}{d t} \in L^{p^{\prime}}\left([s, T], V^{\prime}\right)$. Now by the general fact (see p.164 of [14]) it follows that $v$ is almost everywhere equal to a function belonging to $C([s, T], H)$. Hence by the transformation (3.9) and the continuous property of Ornstein-Uhlenbeck process, $u\left(t, \omega ; s, u_{0}\right)$ is almost everywhere equal to a function belonging to $C([s, T], H)$.

We finally prove the continuity of the mapping $u_{0} \mapsto u\left(t, \omega ; s, u_{0}\right)$ from $H$ into $H$. It suffices to prove that the mapping $v_{0} \mapsto v\left(t, \omega ; s, v_{0}\right)$ is continuous from $H$ into $H$.

Let $v_{1}, v_{2}$ be two different initial values at initial value time $s$, and corresponding solutions be denoted by $v^{1}\left(t, \omega ; s, v_{1}\right)$ and $v^{2}\left(t, \omega ; s, v_{2}\right)$ respectively. Then it follows from (3.14) that

$$
\frac{d}{d t}\left(v^{1}(t)-v^{2}(t)\right)+\bar{A}\left(v^{1}(t), \omega\right)-\bar{A}\left(v^{2}(t), \omega\right)=0,
$$

where $\bar{A}(v(t), \omega)$ is defined in (3.13). Note that

$$
\begin{aligned}
( & \left.\Delta \Phi\left(\Delta v^{1}(t)\right)-\Delta \Phi\left(\Delta v^{2}(t)\right), v^{1}(t)-v^{2}(t)\right) \\
& =\left(\left|\Delta v^{1}(t)\right|^{p-2} \Delta v^{1}(t)-\left|\Delta v^{2}(t)\right|^{p-2} \Delta v^{2}(t), \Delta v^{1}(t)-\Delta v^{2}(t)\right) \\
& =\int_{D}\left(\left|\Delta v^{1}(t)\right|^{p}+\left|\Delta v^{2}(t)\right|^{p}-\left|\Delta v^{1}(t)\right|^{p-2} \Delta v^{1}(t) \Delta v^{2}(t)-\left|\Delta v^{2}(t)\right|^{p-2} \Delta v^{2}(t) \Delta v^{1}(t)\right) d x \\
& \geq \int_{D}\left(\left|\Delta v^{1}(t)\right|^{p}+\left|\Delta v^{2}(t)\right|^{p}-\left|\Delta v^{1}(t)\right|^{p-1}\left|\Delta v^{2}(t)\right|-\left|\Delta v^{2}(t)\right|^{p-1}\left|\Delta v^{1}(t)\right|\right) d x \\
& =\int_{D}\left(\left|\Delta v^{1}(t)\right|^{p-1}-\left|\Delta v^{2}(t)\right|^{p-1}\right)\left(\left|\Delta v^{1}(t)\right|-\left|\Delta v^{2}(t)\right|\right) d x \geq 0 .
\end{aligned}
$$

Because the function $u^{p-1}$ is increasing for $u \geq 0$ and $p \geq 2$, the last inequality in the above proof is correct. Then by a simple computation we find that for fixed $\omega \in \Omega$,

$$
\left(\bar{A}\left(v^{1}(t), \omega\right)-\bar{A}\left(v^{2}(t), \omega\right), v^{1}(t)-v^{2}(t)\right) \geq\left(k_{3}-b z\left(\theta_{t} \omega\right)\right)\left\|v^{1}(t)-v^{2}(t)\right\|_{2}^{2},
$$


where $k_{3}$ is in (3.6). Hence, multiplying (3.15) by $v^{1}(t)-v^{2}(t)$, integrating over $D$, and using (3.16), we get that

$$
\frac{d}{d t}\left\|v^{1}(t)-v^{2}(t)\right\|_{2}^{2}+2\left(k_{3}-b z\left(\theta_{t} \omega\right)\right)\left\|v^{1}(t)-v^{2}(t)\right\|_{2}^{2} \leq 0 .
$$

Using Gronwall's lemma to (3.17) from $s$ to $t$, it yields that

$$
\left\|v^{1}(t)-v^{2}(t)\right\|_{2}^{2} \leq e^{\int_{s}^{t} 2\left(-k_{3}+b z\left(\theta_{\tau} \omega\right)\right) d \tau}\left\|v_{1}-v_{2}\right\|_{2}^{2} .
$$

Then, the continuity of the mapping $v_{0} \mapsto v\left(t, \omega ; s, v_{0}\right)$ from $H$ into $H$ is followed from the contraction property (3.18). This finishes the total proofs of Theorem 3.1.

We now define

$$
S(t, s ; \omega) u_{0}=u\left(t, \omega ; s, u_{0}\right), \quad t \geq s \in \mathbb{R},
$$

with $u_{0}=u(s)$. By the uniqueness part of the solution in Theorem 3.1, we immediately get that $S(t, s ; \omega)$ is a stochastic flow; that is, for every $u_{0} \in H$ and $t \geq r \geq s \in \mathbb{R}$

$$
\begin{aligned}
& S(t, s ; \omega) u_{0}=S(t, r ; \omega) S(r, s ; \omega) u_{0}, \\
& S(t, s ; \omega) u_{0}=S\left(t-s, 0 ; \theta_{s} \omega\right) u_{0} .
\end{aligned}
$$

Hence if we define

$$
\psi(t, \omega) u_{0}=S(t, 0 ; \omega) u_{0}=u\left(t, \omega ; 0, u_{0}\right)
$$

with $u_{0}=u(0)$, then by Theorem $3.1 \psi$ is a continuous RDS associated with System (3.1)(3.3).

We define

$$
\varphi(t, \omega) v_{0}=v\left(t, \omega ; 0, v_{0}\right)=e^{-b z\left(\theta_{t} \omega\right)} \psi(t, \omega) u_{0}=e^{-b z\left(\theta_{t} \omega\right)} u\left(t, \omega ; 0, u_{0}\right)
$$

Then $\varphi$ is a continuous RDS associated with System (3.10)-(3.12), with the following fact

$$
\varphi\left(t, \theta_{-t} \omega\right) v_{0}=v\left(0, \omega ;-t, v_{0}\right) \quad \text { for all } t \geq 0 .
$$

That is to say, $\varphi\left(t, \theta_{-t} \omega\right) v_{0}$ can be interpreted as the position of the trajectory at time 0 , which was in $v_{0}$ at time $-t$ (see [5]).

It is easy to check that $\psi$ possesses a random attractor provided that $\varphi$ possesses a random attractor. Hence in the following we only concentrate on the RDS $\varphi$.

\section{Existence of compact random attractor for RDS}

In this section, we will compute some estimates in space $H=L^{2}(D)$ and $V_{0}=H_{0}^{1}(D)$. Note that in the following $\omega \in \Omega$; the results will hold for $\mathbb{P}$-a.e. $\omega \in \Omega$ and the generic constants $c$ or $c_{i}, i=1,2, \ldots$ are independent of $\lambda>0$ in the context, where $\lambda \in \rho(\Delta)$. 
Lemma 4.1 Suppose that $g$ satisfies (3.4)-(3.6) and $f$ is given in $V^{\prime}$. Then there exist random radii $r_{1}(\omega), r_{2}(\omega)>0$, such that for all $\varrho>0$ there exists $s=s(\omega, \varrho) \leq-1$ such that for all $s \leq s(\omega, \varrho)$ and all $v_{0} \in H$ with $\left\|v_{0}\right\|_{2} \leq \varrho$, the following inequalities hold for $\mathbb{P}$-a.e. $\omega \in \Omega$,

$$
\begin{aligned}
& \left\|v\left(t, \omega ; s, v_{0}\right)\right\|_{2}^{2} \leq r_{1}^{2}(\omega) \quad \text { for all } t \in[-1,0], \\
& \int_{-1}^{0}\left(\left\|\Delta v\left(\tau, \omega ; s, v_{0}\right)\right\|_{p}^{p}+\left\|v\left(\tau, \omega ; s, v_{0}\right)\right\|_{q}^{q}\right) d \tau \leq r_{2}^{2}(\omega),
\end{aligned}
$$

where $v\left(t, \omega ; s, v_{0}\right)$ is the solution to Equation (3.10) with $v_{0}=v(s)$.

Proof For simplicity, we abbreviate $v(t)=: v\left(t, \omega ; s, v_{0}\right)$ for $t \geq s$ with $v_{0}=v(s)$. Multiplying both sides of (3.10) by $v(t)$ and then integrating over $D$, we obtain that

$$
\begin{gathered}
\frac{1}{2} \frac{d}{d t}\|v\|_{2}^{2}+e^{b(p-2) z\left(\theta_{t} \omega\right)}(\Delta \Phi(\Delta v), v)+e^{-b z\left(\theta_{t} \omega\right)} \int_{D} g\left(x, e^{b z\left(\theta_{t} \omega\right)} v\right) v d x \\
=e^{-b z\left(\theta_{t} \omega\right)}(f, v)+b z\left(\theta_{t} \omega\right)\|v\|_{2}^{2}
\end{gathered}
$$

where

$$
\begin{aligned}
& e^{b(p-2) z\left(\theta_{t} \omega\right)}(\Delta \Phi(\Delta v), v)=e^{b(p-2) z\left(\theta_{t} \omega\right)}\|\Delta v\|_{p^{\prime}}^{p}, \\
& e^{-b z\left(\theta_{t}(\omega)\right.} \int_{D} g\left(x, e^{b z\left(\theta_{t} \omega\right)} v\right) v d x \geq k_{1} e^{b(q-2) z\left(\theta_{t}(\omega)\right.}\|v\|_{q}^{q}-e^{-2 b z\left(\theta_{t}(\omega)\right.}\left\|\phi_{1}\right\|_{1}, \\
& e^{-b z\left(\theta_{t}(\omega)\right.}(f, v) \leq e^{-b z\left(\theta_{t} \omega\right)}\|f\|_{p^{\prime}}\|v\|_{p} \leq \frac{1}{2} e^{b(p-2) z\left(\theta_{t} \omega\right)}\|\Delta v\|_{p}^{p}+c e^{-2 b z\left(\theta_{t}(\omega)\right.}\|f\|_{p^{\prime}}^{p^{\prime}}
\end{aligned}
$$

Then by (4.1)-(4.4), we have

$$
\begin{aligned}
& \frac{d}{d t}\|v\|_{2}^{2}+e^{b(p-2) z\left(\theta_{t} \omega\right)}\|\Delta v\|_{p}^{p}+2 k_{1} e^{b(q-2) z\left(\theta_{t} \omega\right)}\|v\|_{q}^{q} \\
& \quad \leq 2 b z\left(\theta_{t} \omega\right)\|v\|_{2}^{2}+2 e^{-2 b z\left(\theta_{t} \omega\right)}\left(c\|f\|_{p^{\prime}}^{p^{\prime}}+\left\|\phi_{1}\right\|_{1}\right) \\
& \quad \leq 2 b z\left(\theta_{t} \omega\right)\|v\|_{2}^{2}+c e^{-2 b z\left(\theta_{t} \omega\right)} .
\end{aligned}
$$

Since $q \geq 2$, then by using Sobolev's embedding inequality and inverse Young's inequality we see that

$$
2 k_{1} e^{b(q-2) z\left(\theta_{t} \omega\right)}\|v\|_{q}^{q} \geq 2 c k_{1} e^{b(q-2) z\left(\theta_{t} \omega\right)}\|v\|_{2}^{q} \geq 2\|v\|_{2}^{2}-2 c e^{-2 b z\left(\theta_{t} \omega\right)} .
$$

Then it follows from (4.5) and (4.6) that

$$
\frac{d}{d t}\|v\|_{2}^{2} \leq\left(-2+2 b z\left(\theta_{t} \omega\right)\right)\|v\|_{2}^{2}+c e^{-2 b z\left(\theta_{t} \omega\right)} .
$$

By employing Gronwall's lemma over interval $[s, t]$ with $t \in[-1,0]$, we find that

$$
\begin{aligned}
\|v(t)\|_{2}^{2} & \leq e^{\int_{s}^{t}\left(-2+2 b z\left(\theta_{\tau}(\omega)\right) d \tau\right.}\|v(s)\|_{2}^{2}+c \int_{s}^{t} e^{-2 b z\left(\theta_{\tau} \omega\right)} e^{\int_{\tau}^{t}\left(-2+2 b z\left(\theta_{\sigma}(\omega)\right) d \sigma\right.} d \tau \\
& =e^{\int_{t}^{0}\left(2-2 b z\left(\theta_{\tau} \omega\right)\right) d \tau}\left(e^{\int_{s}^{0}\left(-2+2 b z\left(\theta_{\tau} \omega\right)\right) d \tau}\|v(s)\|_{2}^{2}\right.
\end{aligned}
$$




$$
\begin{array}{r}
\left.+c \int_{-\infty}^{0} e^{-2 b z\left(\theta_{\tau} \omega\right)+\int_{\tau}^{0} 2 b z\left(\theta_{\sigma} \omega\right) d \sigma+2 \tau} d \tau\right) \\
\leq e^{\int_{-1}^{0}\left(2+2 b\left|z\left(\theta_{\tau} \omega\right)\right|\right) d \tau}\left(e^{\int_{s}^{0}\left(-2+2 b z\left(\theta_{\tau} \omega\right)\right) d \tau} \varrho^{2}\right. \\
\left.+c \int_{-\infty}^{0} e^{-2 b z\left(\theta_{\tau} \omega\right)+\int_{\tau}^{0} 2 b z\left(\theta_{\sigma} \omega\right) d \sigma+2 \tau} d \tau\right)
\end{array}
$$

for $\|v(s)\|_{2}^{2} \leq \varrho^{2}$. By the properties of the Ornstein-Uhlenbeck process, we deduce that

$$
\lim _{s \rightarrow-\infty} e^{\int_{s}^{0}\left(-2+2 b z\left(\theta_{\tau} \omega\right)\right) d \tau} \varrho^{2}=0
$$

and

$$
\int_{-\infty}^{0} e^{-2 b z\left(\theta_{\tau} \omega\right)+\int_{\tau}^{0} 2 b z\left(\theta_{\sigma} \omega\right) d \sigma+2 \tau} d \tau<+\infty
$$

Hence, given every fixed $\varrho>0$ and $\|v(s)\|_{2}^{2} \leq \varrho^{2}$, we can choose $s(\omega, \varrho) \leq-1$, depending only on $\omega$ and $\varrho$, such that for all $s \leq s(\omega, \varrho)$ and $t \in[-1,0]$,

$$
\left\|v\left(t, \omega ; s, v_{0}\right)\right\|_{2}^{2} \leq e^{\int_{-1}^{0}\left(2+2 b\left|z\left(\theta_{\tau} \omega\right)\right|\right) d \tau}\left(1+c \int_{-\infty}^{0} e^{-2 b z\left(\theta_{\tau} \omega\right)+c \int_{\tau}^{0} 2 b z\left(\theta_{\sigma} \omega\right) d \sigma+2 \tau} d \tau\right),
$$

which gives an expression for $r_{1}^{2}(\omega)$. Replacing $t$ by $\tau$ in (4.5) and integrating for $\tau$ over intervals $[-1,0]$, then using (4.11) it yields that for all $s \leq s(\omega, \varrho)$,

$$
\begin{aligned}
& \int_{-1}^{0}\left(e^{b(p-2) z\left(\theta_{\tau} \omega\right)}\left\|\Delta v\left(\tau, \omega ; s, v_{0}\right)\right\|_{p}^{p}+2 k_{1} e^{b(q-2) z\left(\theta_{\tau} \omega\right)}\left\|v\left(\tau, \omega ; s, v_{0}\right)\right\|_{q}^{q}\right) d \tau \\
& \quad \leq 2 b \int_{-1}^{0} z\left(\theta_{\tau} \omega\right)\|v(\tau)\|_{2}^{2} d \tau+c \int_{-1}^{0} e^{-2 b z\left(\theta_{\tau} \omega\right)} d \tau+\|v(-1)\|_{2}^{2} \\
& \quad \leq 2 b r_{1}^{2}(\omega) \int_{-1}^{0} z\left(\theta_{\tau} \omega\right) d \tau+c \int_{-1}^{0} e^{-2 b z\left(\theta_{\tau} \omega\right)} d \tau+r_{1}^{2}(\omega) .
\end{aligned}
$$

Then we have

$$
\begin{aligned}
& \int_{-1}^{0}\left(\left\|\Delta v\left(\tau, \omega ; s, v_{0}\right)\right\|_{p}^{p}+\left\|v\left(\tau, \omega ; s, v_{0}\right)\right\|_{q}^{q}\right) d \tau \\
& \quad \leq m(\omega)\left(2 b r_{1}^{2}(\omega) \int_{-1}^{0} z\left(\theta_{\tau} \omega\right) d \tau+c \int_{-1}^{0} e^{-2 b z\left(\theta_{\tau} \omega\right)} d \tau+r_{1}^{2}(\omega)\right)
\end{aligned}
$$

where

$$
m(\omega)^{-1}=\min \left\{\min _{-1 \leq t \leq 0}\left\{e^{b(p-2) z\left(\theta_{\tau} \omega\right)}\right\}, \min _{-1 \leq t \leq 0}\left\{2 k_{1} e^{b(q-2) z\left(\theta_{\tau} \omega\right)}\right\}\right\} .
$$

Thus the right-hand side of (4.13) gives an expression for $r_{2}^{2}(\omega)$.

In the following, we shall obtain the regularity of the solution to stochastic $p$-Laplaciantype equation. This is the most challenging part in our discussion. Because of the nonlinearity of driven $\Delta \Phi(\Delta u)$ and function $g(x, u)$ in Equation (3.10), it seems difficult to 
derive the $V$-norm estimate as in [14], where the author only deals with a linear case, i.e., $g(x, u)=k u$. So we relax to estimate the solution in a weaker Sobolev $V_{0}=H_{0}^{1}(D)$ with equivalent norms denoted by $\|\nabla v\|_{2}$ for $v \in V_{0}$. Here, just as stated in the introduction, we use the properties of Dirichlet forms for the Laplacian $\Delta$.

Lemma 4.2 Suppose that $g$ satisfies (3.4)-(3.6) and $f$ is given in $V^{\prime}$. Then there exists a random radius $r_{3}(\omega)>0$, such that for all $\varrho>0$ there exists $s=s(\omega, \varrho) \leq-1$ such that for all $s \leq s(\omega, \varrho)$ and all $v_{0} \in H$ with $\left\|v_{0}\right\|_{2} \leq \varrho$, the following inequality holds for $\mathbb{P}$-a.e. $\omega \in \Omega$

$$
\left\|\nabla v\left(t, \omega ; s, v_{0}\right)\right\|_{2}^{2} \leq r_{3}^{2}(\omega) \quad \text { for all } t \in[-1,0]
$$

where $v\left(t, \omega ; s, v_{0}\right)$ is the solution to (3.10) with $v_{0}=v(s)$.

Proof Taking the inner product of (3.10) with $-\lambda \Delta R(\lambda, \Delta) v$ where $\lambda>0$ and $v \in V$, we get that

$$
\begin{aligned}
& -\int_{D} v_{t} \lambda \Delta R(\lambda, \Delta) v d x \\
& =e^{b(p-2) z\left(\theta_{t} \omega\right)} \int_{D} \Delta \Phi(\Delta v) \lambda \Delta R(\lambda, \Delta) v d x \\
& \quad+e^{-b z\left(\theta_{t} \omega\right)} \int_{D} g\left(x, e^{b z\left(\theta_{t} \omega\right)} v\right) \lambda \Delta R(\lambda, \Delta) v d x \\
& \quad-e^{-b z\left(\theta_{t} \omega\right)} \int_{D} f(x) \lambda \Delta R(\lambda, \Delta) v d x-b z\left(\theta_{t} \omega\right) \int_{D} v \lambda \Delta R(\lambda, \Delta) v d x .
\end{aligned}
$$

By the semigroup theory (see [14]) we have

$$
\Delta R(\lambda, \Delta) v=R(\lambda, \Delta) \Delta v=\lambda R(\lambda, \Delta) v-v
$$

for $v \in D(\Delta)$, the domain of Laplacian $\Delta$. We now estimate all terms on the right-hand side of (4.14). Employing (4.15) and integrating by parts, it yields that

$$
\begin{aligned}
\int_{D} \Delta \Phi(\Delta v) \lambda \Delta R(\lambda, \Delta) v= & \lambda \int_{D} \Delta \Phi(\Delta v)(\lambda R(\lambda, \Delta) v-v) d x \\
= & -\lambda \int_{D} \Delta\left(|\Delta v|^{p-2} \Delta v\right) v d x \\
& +\lambda \int_{D} \Delta\left(|\Delta v|^{p-2} \Delta v\right) \lambda R(\lambda, \Delta) v d x \\
= & -\lambda\|\Delta v\|_{p}^{p}+\lambda \int_{D}\left(|\Delta v|^{p-2} \Delta v\right) \lambda \Delta R(\lambda, \Delta) v d x \\
\leq & -\lambda\|\Delta u\|_{p}^{p}+\lambda \int_{D}|\Delta u|^{p-1}|\lambda R(\lambda, \Delta) \Delta v| d x \\
\leq & -\lambda\|\Delta v\|_{p}^{p}+\lambda\|\Delta v\|_{p}^{p-1}\|\lambda R(\lambda, \Delta) \Delta v\|_{p} \\
\leq & -\lambda\|\Delta v\|_{p}^{p}+\lambda\|\Delta v\|_{p}^{p}=0,
\end{aligned}
$$

where we use the contraction property of $\lambda R(\lambda, \Delta)$ on $L^{p}(D)$, i.e.,

$$
\|\lambda R(\lambda, \Delta) \Delta v\|_{p} \leq\|\Delta v\|_{p}
$$


for $\Delta v \in L^{p}(D)$ and every $\lambda>0$. By our assumption (3.5), along with (4.17) for $q$, the second term on the right-hand side of (4.14) is estimated as

$$
\begin{aligned}
& e^{-b z\left(\theta_{t} \omega\right)} \int_{D} g\left(x, e^{b z\left(\theta_{t} \omega\right)} v\right) \lambda \Delta R(\lambda, \Delta) \nu d x \\
& \leq e^{-b z\left(\theta_{t} \omega\right)} \int_{D}\left|g\left(x, e^{b z\left(\theta_{t} \omega\right)} v\right)\right||\lambda R(\lambda, \Delta) \Delta v| d x \\
& \leq e^{-b z\left(\theta_{t} \omega\right)} \int_{D}\left(k_{2} e^{b(q-1) z\left(\theta_{t} \omega\right)}|v|^{q-1}+\phi_{2}(x)\right)|\lambda R(\lambda, \Delta) \Delta v| d x \\
& \leq k_{2} e^{b(q-2) z\left(\theta_{t} \omega\right)}\|v\|_{q}^{q-1}\|\lambda R(\lambda, \Delta) \Delta v\|_{q}+e^{-b z\left(\theta_{t} \omega\right)}\left\|\phi_{2}\right\|_{q^{\prime}}\|\lambda R(\lambda, \Delta) \Delta v\|_{q} \\
& \leq k_{2} e^{b(q-2) z\left(\theta_{t} \omega\right)}\|v\|_{q}^{q-1}\|\Delta v\|_{q}+e^{-b z\left(\theta_{t} \omega\right)}\left\|\phi_{2}\right\|_{q^{\prime}}\|\Delta v\|_{q} \\
& \leq k_{2} e^{b(q-2) z\left(\theta_{t} \omega\right)}\|v\|_{q}^{q}+k_{2} e^{b(q-2) z\left(\theta_{t} \omega\right)}\|\Delta v\|_{q}^{q}+e^{-b z\left(\theta_{t} \omega\right)}\|\Delta v\|_{q}^{q}+e^{-b z\left(\theta_{t} \omega\right)}\left\|\phi_{2}\right\|_{q^{\prime}}^{q^{\prime}}
\end{aligned}
$$

where we employ Young's inequality $a b \leq a^{r}+b^{\frac{r}{r-1}}$ for $r>1$ twice. But, by Sobolev's inequality and Young's inequality, it yields that

$$
\begin{aligned}
k_{2} e^{b(q-2) z\left(\theta_{t} \omega\right)}\|\Delta v\|_{q}^{q} & \leq c e^{b(q-2) z\left(\theta_{t} \omega\right)}\|\Delta v\|_{p}^{q} \\
& =e^{\frac{b q(p-2)}{p} z\left(\theta_{t} \omega\right)}\|\Delta v\|_{p}^{q} \cdot c e^{\frac{2 b(q-p)}{p} z\left(\theta_{t} \omega\right)} \\
& \leq e^{b(p-2) z\left(\theta_{t} \omega\right)}\|\Delta v\|_{p}^{p}+c e^{-2 b z\left(\theta_{t} \omega\right)}
\end{aligned}
$$

and by (4.19) we have

$$
\begin{aligned}
e^{-b z\left(\theta_{t} \omega\right)}\|\Delta v\|_{q}^{q} & =\frac{1}{k_{2}} e^{-b(q-1) z\left(\theta_{t} \omega\right)} \cdot k_{2} e^{b(q-2) z\left(\theta_{t} \omega\right)}\|\Delta v\|_{q}^{q} \\
& \leq \frac{1}{k_{2}} e^{-b(q-1) z\left(\theta_{t} \omega\right)}\left(e^{b(p-2) z\left(\theta_{t} \omega\right)}\|\Delta v\|_{p}^{p}+c e^{-2 b z\left(\theta_{t} \omega\right)}\right) \\
& \leq \frac{1}{k_{2}} e^{b(p-q-1) z\left(\theta_{t} \omega\right)}\|\Delta v\|_{p}^{p}+c e^{-b(q+1) z\left(\theta_{t} \omega\right)}
\end{aligned}
$$

Then by (4.18)-(4.20), there exist positive constants $c$ such that

$$
\begin{aligned}
e^{-b z\left(\theta_{t} \omega\right)} & \int_{D} g\left(x, e^{b z\left(\theta_{t} \omega\right)} v\right) \lambda \Delta R(\lambda, \Delta) v d x \\
\leq & \left(e^{b(p-2) z\left(\theta_{t} \omega\right)}+\frac{1}{k_{2}} e^{b(p-q-1) z\left(\theta_{t} \omega\right)}\right)\|\Delta v\|_{p}^{p} \\
& +k_{2} e^{b(q-2) z\left(\theta_{t} \omega\right)}\|v\|_{q}^{q}+c\left(e^{-b z\left(\theta_{t} \omega\right)}+e^{-2 b z\left(\theta_{t} \omega\right)}+e^{-b(q+1) z\left(\theta_{t} \omega\right)}\right) .
\end{aligned}
$$

For the third term on the right-hand side of (4.14), by (4.17) we see that

$$
\begin{aligned}
-e^{-b z\left(\theta_{t} \omega\right)} \int_{D} f(x) \lambda \Delta R(\lambda, \Delta) \nu d x & \leq e^{-b z\left(\theta_{t} \omega\right)}\|f\|_{p^{\prime}}\|\lambda R(\lambda, \Delta) \Delta v\|_{p} \\
& \leq e^{-b z\left(\theta_{t} \omega\right)}\|f\|_{p^{\prime}}\|\Delta v\|_{p} \\
& \leq e^{b(p-2) z\left(\theta_{t} \omega\right)}\|\Delta v\|_{p}^{p}+e^{-2 b z\left(\theta_{t} \omega\right)}\|f\|_{p^{\prime}}^{p^{\prime}}
\end{aligned}
$$


On the other hand, by (4.15) and the Dirichlet forms $\&$ (2.3), we have

$$
-\int_{D} v_{t} \lambda \Delta R(\lambda, \Delta) v d x=\mathcal{E}^{(\lambda)}\left(v, v_{t}\right), \quad-\int_{D} v \lambda \Delta R(\lambda, \Delta) \nu d x=\mathcal{E}^{(\lambda)}(v, v) .
$$

Then it follows from (4.14), (4.16) and (4.21)-(4.23) that

$$
\mathcal{E}^{(\lambda)}\left(\nu, v_{t}\right) \leq p_{1}\left(\theta_{t} \omega\right)\|\Delta v\|_{p}^{p}+p_{2}\left(\theta_{t} \omega\right)\|v\|_{q}^{q}+p_{3}\left(\theta_{t} \omega\right)+b z\left(\theta_{t} \omega\right) \mathcal{E}^{(\lambda)}(v, v),
$$

where

$$
\begin{aligned}
& p_{1}\left(\theta_{t} \omega\right)=2 e^{b(p-2) z\left(\theta_{t} \omega\right)}+\frac{1}{k_{2}} e^{b(p-q-1) z\left(\theta_{t} \omega\right)}, \\
& p_{2}\left(\theta_{t} \omega\right)=k_{2} e^{b(q-2) z\left(\theta_{t} \omega\right)}, \\
& p_{3}\left(\theta_{t} \omega\right)=c\left(e^{-b z\left(\theta_{t} \omega\right)}+e^{-2 b z\left(\theta_{t} \omega\right)}+e^{-b(q+1) z\left(\theta_{t} \omega\right)}\right)
\end{aligned}
$$

and $c$ is a positive constant independent of $\lambda$. So taking limit on both sides of (4.24) for $\lambda \rightarrow \infty$ and associating with (2.2) and (2.4), we deduce that

$$
\frac{1}{2} \frac{d}{d t}\|\nabla v(t)\|_{2}^{2} \leq p_{1}\left(\theta_{t} \omega\right)\|\Delta v\|_{p}^{p}+p_{2}\left(\theta_{t} \omega\right)\|v\|_{q}^{q}+b z\left(\theta_{t} \omega\right)\|\nabla v(t)\|_{2}^{2}+p_{3}\left(\theta_{t} \omega\right)
$$

Replacing $t$ by $\tau$ in (4.25) and integrating $\tau$ from $s$ to $t(-1 \leq s \leq t \leq 0)$, it yields that

$$
\begin{aligned}
\|\nabla v(t)\|_{2}^{2} \leq & 2 \int_{s}^{t} p_{1}\left(\theta_{\tau} \omega\right)\|\Delta v(\tau)\|_{p}^{p} d \tau+2 \int_{s}^{t} p_{2}\left(\theta_{\tau} \omega\right)\|v(\tau)\|_{q}^{q} d \tau \\
& +2 \int_{s}^{t} p_{3}\left(\theta_{\tau} \omega\right) d \tau+2 b \int_{s}^{t} z\left(\theta_{\tau} \omega\right)\|\nabla v(\tau)\|_{2}^{2} d \tau+\|\nabla v(s)\|_{2}^{2} \\
\leq & 2 \int_{-1}^{0} p_{1}\left(\theta_{\tau} \omega\right)\|\Delta v(\tau)\|_{p}^{p} d \tau+2 \int_{-1}^{0} p_{2}\left(\theta_{\tau} \omega\right)\|v(\tau)\|_{q}^{q} d \tau \\
& +2 \int_{-1}^{0} p_{3}\left(\theta_{\tau} \omega\right) d \tau+2 b \int_{-1}^{0} z\left(\theta_{\tau} \omega\right)\|\nabla v(\tau)\|_{2}^{2} d \tau+\|\nabla v(s)\|_{2}^{2} .
\end{aligned}
$$

Put

$$
M(\omega)=\max \left\{\max _{-1 \leq \tau \leq 0}\left\{p_{1}\left(\theta_{\tau} \omega\right)\right\}, \max _{-1 \leq \tau \leq 0}\left\{p_{2}\left(\theta_{\tau} \omega\right)\right\}, \max _{-1 \leq \tau \leq 0}\left\{p_{3}\left(\theta_{\tau} \omega\right)\right\}, \max _{-1 \leq \tau \leq 0}\left\{z\left(\theta_{\tau} \omega\right)\right\}\right\} .
$$

Then by Lemma 4.1, (4.26) reads

$$
\|\nabla v(t)\|_{2}^{2} \leq 2 M(\omega) r_{2}^{2}(\omega)+2 M(\omega)+2 b M(\omega) \int_{-1}^{0}\|\nabla v(\tau)\|_{2}^{2} d \tau+\|\nabla v(s)\|_{2}^{2} .
$$

Integrating (4.27) for $s$ over intervals $[-1,0]$, we have

$$
\|\nabla v(t)\|_{2}^{2} \leq 2 M(\omega) r_{2}^{2}(\omega)+2 M(\omega)+(2 b M(\omega)+1) \int_{-1}^{0}\|\nabla v(\tau)\|_{2}^{2} d \tau
$$


for all $t \in[-1,0]$. By Poincare's inequality and Young's inequality, there exists positive constant $c$ such that

$$
\|\nabla v(s)\|_{2}^{2} \leq c\|\Delta v(s)\|_{2}^{2} \leq\|\Delta v(s)\|_{p}^{p}+c .
$$

Hence by using Lemma 4.1 again, along with (4.29), it follows from (4.28) that

$$
\|\nabla v(t)\|_{2}^{2} \leq 2 M(\omega) r_{2}^{2}(\omega)+2 M(\omega)+(2 b M(\omega)+1) r_{2}(\omega)+c,
$$

with $t \in[-1,0]$, which gives an expression for $r_{3}^{2}(\omega)$. This completes the proof.

By Theorem 2.3 and Lemma 4.2, we have obtained our main result in this section.

Theorem 4.3 Assume that $g$ satisfies (3.4)-(3.6) and $f$ is given in $V^{\prime}$. Then the RDS $\varphi(t, \omega)$ generated by System (3.10)-(3.12) possesses a random attractor $\{\mathcal{A}(\omega)\}_{\omega \in \Omega}$ defined by

$$
\mathcal{A}(\omega)=\overline{\bigcup_{B \in \mathcal{B}(H)} \bigcap_{s \geq 0} \overline{\bigcup_{t \geq s} \varphi\left(t, \theta_{-t} \omega\right) B}}
$$

where $\mathcal{B}(H)$ denotes all the bounded subsets of $H$ and the closure is the $H$-norm.

\section{The single point attractor}

In this section, we consider a special case, that is, $k_{3}>0$ in (3.6), in which case we find that the random attractor is just composed of a single point. This shows that System (3.10)(3.12) possesses an unique stationary solution for every given initial value in the space $H$. We begin with a lemma.

Lemma 5.1 Assume that $g$ satisfies (3.4)-(3.6) and $f$ is given in $V^{\prime}, k_{3}>0$. Then for $s_{1} \leq$ $s_{2} \leq t$ and $v\left(s_{1}\right), v\left(s_{2}\right) \in H$, there exists a positive constant $k<k_{3}$ such that

$$
\begin{aligned}
& \left\|v\left(t, \omega ; s_{1}, v\left(s_{1}\right)\right)-v\left(t, \omega ; s_{2}, v\left(s_{2}\right)\right)\right\|_{2}^{2} \\
& \leq 2\left\{\left\|v\left(s_{2}\right)\right\|_{2}^{2} e^{\int_{s_{2}}^{0}\left(-k_{3}-b z\left(\theta_{\tau} \omega\right)\right) d \tau}+\left(e^{\int_{s_{1}}^{0}\left(-k+2 b z\left(\theta_{\tau} \omega\right)\right) d \tau}\left\|v\left(s_{1}\right)\right\|_{2}^{2}\right.\right. \\
& \left.\left.\quad+c \int_{-\infty}^{0} e^{-2 b z\left(\theta_{\tau}(\omega)+c \int_{\tau}^{0}\left(-k+2 b z\left(\theta_{\sigma} \omega\right)\right) d \sigma\right.} d \tau\right) e^{\int_{s_{2}}^{0}\left(k-k_{3}-b z\left(\theta_{\tau} \omega\right)\right) d \tau}\right\} e^{-\int_{0}^{t}\left(k_{3}-b z\left(\theta_{\tau} \omega\right)\right) d \tau} .
\end{aligned}
$$

In particular, for each fixed $t \in \mathbb{R}$ and $\omega \in \Omega$ there exists a single point $\varsigma_{t}(\omega)$ in $H$ such that

$$
\lim _{s \rightarrow-\infty} v(t, \omega ; s, v(s))=\varsigma_{t}(\omega)
$$

for every $v(s)$ belonging to the bounded subset $B$ of $H$. Furthermore, the convergence in (5.1) is uniform with respect to all $v(s) \in B$.

Proof Let $v\left(t, \omega ; s_{i}, v\left(s_{i}\right)\right)$ be the solutions to (3.10) with initial values $v\left(s_{i}\right) \in H, i=1,2$. Then we can deduce from (3.14) that

$$
\begin{aligned}
& \frac{d}{d t}\left(v\left(t, \omega ; s_{1}, v\left(s_{1}\right)\right)-v\left(t, \omega ; s_{2}, v\left(s_{2}\right)\right)\right) \\
& \quad+\bar{A}\left(v\left(t, \omega ; s_{1}, v\left(s_{1}\right)\right), \omega\right)-\bar{A}\left(v\left(t, \omega ; s_{2}, v\left(s_{2}\right)\right), \omega\right)=0 .
\end{aligned}
$$


Multiplying (5.2) by $v\left(t, \omega ; s_{1}, v\left(s_{1}\right)\right)-v\left(t, \omega ; s_{2}, v\left(s_{2}\right)\right)$, integrating over $D$ and using (3.16), we find that

$$
\begin{aligned}
& \frac{d}{d t}\left\|v\left(t, \omega ; s_{1}, v\left(s_{1}\right)\right)-v\left(t, \omega ; s_{2}, v\left(s_{2}\right)\right)\right\|_{2}^{2} \\
& \quad+\left(k_{3}-b z\left(\theta_{t} \omega\right)\right)\left\|v\left(t, \omega ; s_{1}, v\left(s_{1}\right)\right)-v\left(t, \omega ; s_{2}, u\left(s_{2}\right)\right)\right\|_{2}^{2} \leq 0 .
\end{aligned}
$$

Now, applying Gronwall's lemma to (5.3) from $s_{2}$ to $t$, it yields that

$$
\begin{aligned}
& \left\|v\left(t, \omega ; s_{1}, v\left(s_{1}\right)\right)-v\left(t, \omega ; s_{2}, v\left(s_{2}\right)\right)\right\|_{2}^{2} \\
& \quad \leq\left\|v\left(s_{2}, \omega ; s_{1}, v\left(s_{1}\right)\right)-v\left(s_{2}\right)\right\|_{2}^{2} e^{-\int_{s_{2}}^{t}\left(k_{3}-b z\left(\theta_{\tau} \omega\right)\right) d \tau} \\
& \quad \leq 2\left(\left\|v\left(s_{2}, \omega ; s_{1}, v\left(s_{1}\right)\right)\right\|_{2}^{2}+\left\|v\left(s_{2}\right)\right\|_{2}^{2}\right) e^{-\int_{s_{2}}^{t}\left(k_{3}-b z\left(\theta_{\tau} \omega\right)\right) d \tau} \\
& \quad=2 e^{-\int_{0}^{t}\left(k_{3}-b z\left(\theta_{\tau} \omega\right)\right) d \tau}\left(\left\|v\left(s_{2}, \omega ; s_{1}, v\left(s_{1}\right)\right)\right\|_{2}^{2}+\left\|v\left(s_{2}\right)\right\|_{2}^{2}\right) e^{-\int_{s_{2}}^{0}\left(k_{3}-b z\left(\theta_{\tau} \omega\right)\right) d \tau} .
\end{aligned}
$$

We then estimate $\left\|v\left(s_{2}, \omega ; s_{1}, v\left(s_{1}\right)\right)\right\|_{2}^{2}$. By (4.5) we have

$$
\begin{aligned}
& \frac{d}{d t}\left\|v\left(t, \omega ; s_{1}, v\left(s_{1}\right)\right)\right\|_{2}^{2}+2 k_{1} e^{b(q-2) z\left(\theta_{t} \omega\right)}\left\|v\left(t, \omega ; s_{1}, v\left(s_{1}\right)\right)\right\|_{q}^{q} \\
& \quad \leq 2 b z\left(\theta_{t} \omega\right)\left\|v\left(t, \omega ; s_{1}, v\left(s_{1}\right)\right)\right\|_{2}^{2}+c e^{-2 b z\left(\theta_{t} \omega\right)} .
\end{aligned}
$$

By the Sobolev's embedding inequality and the inverse Young's inequality, we can choose $0<k<k_{3}$ such that

$$
\begin{aligned}
2 k_{1} e^{b(q-2) z\left(\theta_{t} \omega\right)}\left\|v\left(t, \omega ; s_{1}, v\left(s_{1}\right)\right)\right\|_{q}^{q} & \geq 2 c k_{1} e^{b(q-2) z\left(\theta_{t} \omega\right)}\left\|v\left(t, \omega ; s_{1}, v\left(s_{1}\right)\right)\right\|_{2}^{q} \\
& \geq k\left\|v\left(t, \omega ; s_{1}, v\left(s_{1}\right)\right)\right\|_{2}^{2}-c e^{-2 b z\left(\theta_{t} \omega\right)}
\end{aligned}
$$

So by (5.5) and (5.6) we get that

$$
\frac{d}{d t}\left\|v\left(t, \omega ; s_{1}, v\left(s_{1}\right)\right)\right\|_{2}^{2} \leq\left(-k+2 b z\left(\theta_{t} \omega\right)\right)\left\|v\left(t, \omega ; s_{1}, v\left(s_{1}\right)\right)\right\|_{2}^{2}+c e^{-2 b z\left(\theta_{t} \omega\right)}
$$

Using Gronwall's lemma to (5.7) from $s_{1}$ to $s_{2}$ with $s_{1} \leq s_{2} \leq 0$, we get that

$$
\begin{aligned}
& \left\|v\left(s_{2}, \omega ; s_{1}, v\left(s_{1}\right)\right)\right\|_{2}^{2} \\
& \leq\left\|v\left(s_{1}\right)\right\|_{2}^{2} e^{\int_{s_{1}}^{s_{2}}\left(-k+2 b z\left(\theta_{\tau} \omega\right)\right) d \tau}+c \int_{s_{1}}^{s_{2}} e^{-2 b z\left(\theta_{\tau} \omega\right)+\int_{\tau}^{s_{2}}\left(-k+2 b z\left(\theta_{\sigma} \omega\right)\right) d \sigma} d \tau \\
& =e^{\int_{s_{2}}^{0}\left(k-2 b z\left(\theta_{\tau} \omega\right)\right) d \tau}\left(e^{\int_{s_{1}}^{0}\left(-k+2 b z\left(\theta_{\tau} \omega\right)\right) d \tau}\left\|v\left(s_{1}\right)\right\|_{2}^{2}\right. \\
& \left.\quad+c \int_{s_{2}}^{s_{1}} e^{-2 b z\left(\theta_{\tau} \omega\right)+\int_{\tau}^{0}\left(-k+2 b z\left(\theta_{\sigma} \omega\right)\right) d \sigma} d \tau\right) \\
& \leq e^{\int_{s_{2}}^{0}\left(k-2 b z\left(\theta_{\tau} \omega\right)\right) d \tau}\left(e^{\int_{s_{1}}^{0}\left(-k+2 b z\left(\theta_{\tau} \omega\right)\right) d \tau}\left\|v\left(s_{1}\right)\right\|_{2}^{2}+c \int_{-\infty}^{0} e^{-2 b z\left(\theta_{\tau} \omega\right)+\int_{\tau}^{0}\left(-k+2 b z\left(\theta_{\sigma} \omega\right)\right) d \sigma} d \tau\right) .
\end{aligned}
$$

Similar to the argument of (4.10), we know that the integral in the last term on the righthand side of (5.8) is convergent. Hence, it follows from (5.8) and (5.4) that for every 
fixed $t \in \mathbb{R}$,

$$
\begin{aligned}
& \left\|v\left(t, \omega ; s_{1}, v\left(s_{1}\right)\right)-v\left(t, \omega ; s_{2}, v\left(s_{2}\right)\right)\right\|_{2}^{2} \\
& \leq 2\left\{\left\|v\left(s_{2}\right)\right\|_{2}^{2} e^{\int_{s_{2}}^{0}\left(-k_{3}+b z\left(\theta_{\tau}(\omega)\right) d \tau\right.}+\left(e^{\int_{s_{1}}^{0}\left(-k+2 b z\left(\theta_{\tau} \omega\right)\right) d \tau}\left\|v\left(s_{1}\right)\right\|_{2}^{2}\right.\right. \\
& \left.\left.\quad+c \int_{-\infty}^{0} e^{-2 b z\left(\theta_{\tau} \omega\right)+c \int_{\tau}^{0}\left(-k+2 b z\left(\theta_{\sigma} \omega\right)\right) d \sigma} d \tau\right) e^{\int_{s_{2}}^{0}\left(k-k_{3}-b z\left(\theta_{\tau} \omega\right)\right) d \tau}\right\} e^{-\int_{0}^{t}\left(k_{3}-b z\left(\theta_{\tau} \omega\right)\right) d \tau} .
\end{aligned}
$$

So for every bounded subset $B$ of $H$ and $v\left(s_{1}\right), v\left(s_{2}\right) \in B$, it follows from (5.9) that

$$
\left\|v\left(t, \omega ; s_{1}, v\left(s_{1}\right)\right)-v\left(t, \omega ; s_{2}, v\left(s_{2}\right)\right)\right\|_{2}^{2} \rightarrow 0 \quad \text { as } s_{1}, s_{2} \rightarrow-\infty,
$$

since by the properties of the Ornstein-Uhlenbeck process, we have

$$
\lim _{s_{2} \rightarrow-\infty}\left\|v\left(s_{2}\right)\right\|_{2}^{2} e^{\int_{s_{2}}^{0}\left(-k_{3}+b z\left(\theta_{\tau} \omega\right)\right) d \tau}=0
$$

and

$$
\lim _{s_{1} \rightarrow-\infty}\left\|v\left(s_{1}\right)\right\|_{2}^{2} e^{\int_{s_{1}}^{0}\left(-k+b z\left(\theta_{\tau} \omega\right)\right) d \tau}=0
$$

and

$$
\lim _{s_{2} \rightarrow-\infty}\left(\int_{-\infty}^{0} e^{-2 b z\left(\theta_{\tau} \omega\right)+\int_{\tau}^{0}\left(-k+2 b z\left(\theta_{\sigma} \omega\right)\right) d \sigma} d \tau\right) e^{\int_{s_{2}}^{0}\left(k-k_{3}-b z\left(\theta_{\tau} \omega\right)\right) d \tau}=0
$$

Moreover, the convergence in (5.11)-(5.13) is uniform with respect to $v\left(s_{1}\right), v\left(s_{2}\right)$ belonging to every bounded subset of $H$. Then (5.10) implies that for fixed $t \in \mathbb{R}, v(t, \omega ; s, v(s))$ is a Cauchy sequence in $H$ with respect to $s \rightarrow-\infty$. Therefore, by the completeness of $H$, for every fixed $t \in \mathbb{R}$ and $\omega \in \Omega, v(t, \omega ; s, v(s))$ has a limit in $H$ denoted by $\varsigma_{t}(\omega)$, i.e.,

$$
\lim _{s \rightarrow-\infty} v(t, \omega ; s, v(s))=\varsigma_{t}(\omega) .
$$

Theorem 5.2 Assume that $g$ satisfies (3.4)-(3.6) and $f$ is given in $V^{\prime}, k_{3}>0$. Then the $\operatorname{RDS} \varphi(t, \omega)$ generated by the solution to (3.10)-(3.12) possesses a single point attractor $\{\mathcal{A}(\omega)\}_{\omega \in \Omega}$, i.e., there exists a single point $\varsigma_{0}(\omega)$ in $H$ such that

$$
\mathcal{A}(\omega)=\left\{\varsigma_{0}(\omega)\right\} .
$$

Proof Put

$$
\bar{S}(t, s ; \omega)=v(t, \omega ; s, v(s)) .
$$

Then $\bar{S}(t, s ; \omega)$ is a stochastic flow associated with System (3.10)-(3.12) and the RDS $\varphi(t, \omega)=\bar{S}(t, 0 ; \omega)$. By Lemma 5.1 we define

$$
\varsigma_{0}(\omega)=\lim _{s \rightarrow-\infty} \bar{S}(0, s ; \omega) v_{0},
$$


where $v_{0}=v(s)$. Then we need to prove that $\{\mathcal{A}(\omega)\}_{\omega \in \Omega}=\left\{\varsigma_{0}(\omega)\right\}_{\omega \in \Omega}$ is a compact attractor. It is obvious that $\left\{\varsigma_{0}(\omega)\right\}_{\omega \in \Omega}$ is a compact random set. Hence by Definition 2.2 it suffices to prove the invariance and attracting property for $\left\{\varsigma_{0}(\omega)\right\}_{\omega \in \Omega}$. Since by the continuity of $\varphi(t, \omega)$ and the flow properties of $\bar{S}(t, s ; \omega)$, we have

$$
\begin{aligned}
\varphi(t, \omega) \zeta_{0}(\omega) & =\varphi(t, \omega) \lim _{s \rightarrow-\infty} \bar{S}(0, s ; \omega) v_{0}=\lim _{s \rightarrow-\infty} \varphi(t, \omega) \bar{S}(0, s ; \omega) v_{0} \\
& =\lim _{s \rightarrow-\infty} \bar{S}(t, 0 ; \omega) \bar{S}(0, s ; \omega) v_{0}=\lim _{s \rightarrow-\infty} \bar{S}(t, s ; \omega) v_{0} \\
& =\lim _{s \rightarrow-\infty} \bar{S}\left(t-s, 0 ; \theta_{s} \omega\right) v_{0}=\lim _{s \rightarrow-\infty} \bar{S}\left(0, s-t ; \theta_{t} \omega\right) v_{0}=\varsigma_{0}\left(\theta_{t} \omega\right)
\end{aligned}
$$

That is to say, $\varphi(t, \omega) \mathcal{A}(\omega)=\mathcal{A}\left(\theta_{t} \omega\right)$. On the other hand, by the uniform convergence of (5.1), it follows from (3.22) that for every bounded subset $B \subset H$,

$$
\begin{aligned}
\operatorname{dist}\left(\varphi\left(t, \theta_{-t} \omega\right) B, \mathcal{A}(\omega)\right) & =\sup _{v_{0} \in B}\left\|\varphi\left(t, \theta_{-t} \omega\right) v_{0}-\varsigma_{0}(\omega)\right\|_{2} \\
& =\sup _{v_{0} \in B}\left\|\bar{S}(0,-t, \omega) v_{0}-\varsigma_{0}(\omega)\right\|_{2} \rightarrow 0
\end{aligned}
$$

as $t \rightarrow+\infty$. This shows that $\{\mathcal{A}(\omega)\}_{\omega \in \Omega}$ is an attracting set, and thus we complete the proof.

\section{Competing interests}

The author declares that he has no competing interests.

\section{Author's contributions}

ZW carried out all studies in this article.

\section{Acknowledgements}

The author is indebted to the referee for giving some valuable suggestions that improved the presentations of this article. This work was supported by the China NSF Grant (no. 10871217), the Science and Technology Funds of Chongqing Educational Commission (no. KJ120703), the Fundamental Funds of the Central Universities (no. XDJK2009C100) and the Doctor Funds of Southwest University (no. SWU111068).

Received: 28 December 2011 Accepted: 13 April 2012 Published: 22 June 2012

\section{References}

1. Arnold, L: Random Dynamical Systems. Springer, Berlin (1998)

2. Bates, PW, Lu, K, Wang, B: Random attractors for stochastic reaction-diffusion equations on unbounded domains. J Differential Equations 246, 845-869 (2009). doi:10.1016/j.jde.2008.05.017

3. Chen, G, Zhong, C: Uniform attractors for non-autonomous p-Laplacian equations. Nonlinear Anal. 68(11), 3349-3363 (2008). doi:10.1016/j.na.2007.03.025

4. Chueshov, I: Monotone Random Systems Theory and Applications. Springer, Berlin (2002)

5. Crauel, H, Debussche, A, Flandoli, F: Random attractors. J. Dyn. Differ. Equ. 9(2), 307-341 (1997). doi:10.1007/BF02219225

6. Crauel, H, Flandoli, F: Attractors for random dynamical systems. Probab. Theory Relat. Fields 100(3), 365-393 (1994). doi:10.1007/BF01193705

7. Fan, X: Attractors for a damped stochastic wave equation of Sine-Gordon type with sublinear multiplicative noise. Stoch. Anal. Appl. 24, 767-793 (2006). doi:10.1080/07362990600751860

8. Fukushima, M, Oshima, Y, Takeda, M: Dirichlet Forms and Symmetric Markov Processes. Walter de Gruyter, Berlin (1994)

9. Li, Y, Guo, B: Random attractors for quasi-continuous random dynamical systems and applications to stochastic reaction-diffusion equations. J. Differ. Equ. 245(7), 1775-1800 (2008). doi:10.1016/j.jde.2008.06.031

10. Li, J, Li, Y, Wang, B: Random attractors of reaction-diffusion equations with multiplicative noise in $L^{p}$. Appl. Math. Comput. 215, 3399-3407 (2010). doi:10.1016/j.amc.2009.10.033

11. Pazy, A: Semigroups of Linear Operators and Applications to Partial Differential Equations. Springer, New York (1983)

12. Prévôt, C, Röckner, M: A Concise Course on Stochastic Partial Differential Equations. Lecture Notes in Mathematics, vol. 1905, Springer, Berlin (2007)

13. Schmalfuss, B: Backward cocycle and attractors of stochastic differential equations, In: Reitmann V, Riedrich, $T$, Koksch, N (eds.), International Seminar on Applied Mathematics-Nonlinear Dynamics: Attractor Approximation and Global Behavior, Technische Universität, Dresden (1992) pp. 185-192 
14. Temam, R: Infinite-Dimensional Dynamical Systems in Mechanics and Physics. Springer, New York (1997)

15. Wang, B: Random attractors for the stochastic FitzHugh-Nagumo system on unbounded domains. Nonlinear Anal. 71, $2811-2828$ (2009). doi:10.1016/j.na.2009.01.131

16. Wang, B: Asymptotic behavior of stochastic wave equations with critical exponents on $\mathbb{R}^{3}$. Trans. Am. Math. Soc. 363 , 3639-3663 (2011). doi:10.1090/S0002-9947-2011-05247-5

17. Wang, G, Guo, B, Li, Y: The asymptotic behavior of the stochastic Ginzburg-Landau equation with additive noise. Appl. Math. Comput. 198(2), 849-857 (2008). doi:10.1016/j.amc.2007.09.029

18. Wang, Z, Zhou, S: Random attractors for stochastic reaction-diffusion equations with multiplicative noise on unbounded domains. J. Math. Anal. Appl. 384(1), 160-172 (2011). doi:10.1016/j.jmaa.2011.02.082

19. Yang, M, Sun, C, Zhong, C: Existence of a global attractor for a $p$-Laplacian equation in $\mathbb{R}^{n}$. Nonlinear Anal. 66(1), 1-13 (2007). doi:10.1016/j.na.2005.11.004

20. Yang, M, Sun, C, Zhong, C: Global attractor for p-Laplacian equation. J. Math. Anal. Appl. 327(2), 1130-1142 (2007). doi:10.1016/j.jmaa.2006.04.085

21. Zhang, Q: Random attractors for a Ginzburg-Landau equation with additive noise. Chaos Solitons Fractals 39(1), 463-472 (2009). doi:10.1016/j.chaos.2007.03.001

22. Zhao, C, Duan, J: Random attractor for the Ladyzhenskaya model with additive noise. J. Math. Anal. Appl. 362(1), 241-251 (2010). doi:10.1016/j.jmaa.2009.08.050

23. Zhao, W, Li, Y: Existence of random attractor for a p-Laplacian-type equation with additive noise. Abstr. Appl. Anal. vol 2011, Article ID 616451 (2011). doi:10.1155/2011/616451

24. Zhao, W, Li, Y: Asymptotic behavior of two-dimensional stochastic magneto-hydrodynamics equations with additive noises. J. Math. Phys. 52, 072701 (2011). doi:10.1063/1.3614884

25. Zhao, $W, L i, Y:\left(L^{2}, L^{P}\right)$-random attractors for stochastic reaction-diffusion equation on unbounded domains. Nonlinear Anal. 75, 485-502 (2012). doi:10.1016/j.na.2011.08.050

doi:10.1186/1687-2770-2012-61

Cite this article as: Zhao: Asymptotic behavior of stochastic $p$-Laplacian-type equation with multiplicative noise. Boundary Value Problems 2012 2012:61.

\section{Submit your manuscript to a SpringerOpen ${ }^{\circ}$ journal and benefit from:}

- Convenient online submission

- Rigorous peer review

Immediate publication on acceptance

- Open access: articles freely available online

- High visibility within the field

- Retaining the copyright to your article 